\title{
Saccade-Related Information in the Superior Temporal Motion Complex: Quantitative Functional Mapping in the Monkey
}

\author{
Sophia Bakola, ${ }^{1}$ Georgia G. Gregoriou, ${ }^{1}$ Adonis K. Moschovakis, ${ }^{1,2}$ Vassilis Raos, ${ }^{1,2}$ and Helen E. Savaki ${ }^{1,2}$ \\ ${ }^{1}$ Department of Basic Sciences, Faculty of Medicine, University of Crete, 71003 Iraklion, Crete, Greece, and ${ }^{2}$ Institute of Applied and Computational \\ Mathematics, Foundation for Research and Technology-Hellas, 71110 Heraklion, Crete, Greece
}

\begin{abstract}
Although the role of the motion complex [cortical areas middle temporal (V5/MT), medial superior temporal (MST), and fundus of the superior temporal (FST)] in visual motion and smooth-pursuit eye movements is well understood, little is known about its involvement in rapid eye movements (saccades). To address this issue, we used the quantitative ${ }^{14} \mathrm{C}$-deoxyglucose method to obtain functional maps of the cerebral cortex lying in the superior temporal sulcus of rhesus monkeys executing saccades to visual targets and saccades to memorized targets in complete darkness. Fixational effects were observed in MT-foveal, FST, the anterior part of V4-transitional (V4t), and temporal-occipital areas. Saccades to memorized targets activated areas V5/MT, MST, and V4t, which were also activated for saccades to visual targets. Regions activated in the light and in the dark overlapped extensively. In addition, saccades to visual targets activated areas FST and the intermediate part of the polysensory temporal-parietal-occipital area. Cortical activity related to visually guided saccades could be explained, at least in part, by visual motion. Because only oculomotor signals can account for the equally robust activations induced by memory saccades in complete darkness, we suggest that areas V5/MT, MST, and V4t receive and/or process saccade-related oculomotor information.
\end{abstract}

Key words: saccades; temporal cortex; motion complex; MT; MST; FST; V4; V5; TPO; TEO

\section{Introduction}

Until recently, the two types of eye movements responsible for conjugate shifts of the line of sight (rapid or saccadic and slow or smooth pursuit) were thought to be controlled by different oculomotor circuits. The superior colliculus (SC) in the midbrain, the frontal eye field (FEF) and the supplementary eye field (SEF) in the frontal lobe, and the lateral intraparietal area (LIP) in the parietal lobe are of particular importance for saccades. In contrast, it is the motion complex (MC) in the temporal lobe that is of particular importance when it comes to pursuit. Skepticism toward such a dichotomy was raised by more recent evidence suggesting that the same networks and, in some cases, the same neurons control both pursuit and saccades (Krauzlis, 2004). For example, the rostral portion of the SC is involved not only in small saccades and fixation but also in pursuit eye movements (Krauzlis et al., 1997). Also, the involvement of the saccaderelated cortical areas FEF, SEF, and LIP in smooth pursuit has been documented extensively (Heinen, 1995; Bremmer et al.,

Received Sept. 27, 2006; revised Dec. 7, 2006; accepted Dec. 25, 2006

This work was supported by the European Union (FP6 Grant IST-027574) and the General Secretariat of Research and Technology, Hellas (Grant 01ED111). The financial support of "IATRIKO KRITIS" is also gratefully acknowledged. We thank Henry Kennedy for advice regarding histology and Maria Kefalogianni for technical assistance. This work satisfies in part the requirements for awarding a PhD in the "Brain and Mind Sciences" to Sophia Bakola.

Correspondence should be addressed to Helen E. Savaki, University of Crete, P.0. Box 2208, 71003 Iraklion, Crete, Greece. E-mail: savaki@med.uoc.gr.

DOI:10.1523/JNEUROSCI.4224-06.2007

Copyright $\odot 2007$ Society for Neuroscience $\quad 0270-6474 / 07 / 272224-06 \$ 15.00 / 0$
1997; Tanaka and Lisberger, 2002). On the other hand, involvement of the pursuit-related superior temporal MC in saccades remains doubtful. The $\mathrm{MC}$ is located in the caudal (dorsal) portion of the superior temporal sulcus (STs) and consists of the middle temporal visual area (V5/MT), situated in the posterior (lower) bank (Zeki, 1974; Maunsell and Van Essen, 1983), the medial superior temporal area (MST) in the anterior (upper) bank, and the fundus of the superior temporal area (FST) (Desimone and Ungerleider, 1986; Boussaoud et al., 1990). To evaluate the participation of any of these areas in saccades, we used the ${ }^{14} \mathrm{C}$-deoxyglucose $\left({ }^{14} \mathrm{C}-\mathrm{DG}\right)$ method to map the distribution of cortical activity patterns in monkeys executing saccades to visual targets or saccades to memorized locations in the dark.

\section{Materials and Methods}

Behavioral tasks. Six head-fixed adult female monkeys (Macaca mulatta) weighing between 3 and $5 \mathrm{~kg}$ were used. Experiments were approved by the institutional animal use committee in accordance with the European Council Directive 86/609/EEC. A detailed description of surgical procedures and eye position recording was reported previously (Moschovakis et al., 2001). Monkeys were seated in a primate chair $23 \mathrm{~cm}$ from a video monitor. Visual targets were red spots of $1.5^{\circ}$ diameter. Monkeys executing visually guided saccades $(\mathrm{Sl})$ and memorized saccades in complete darkness (Sd) were trained to hold eye position within circular windows of 2.5 and $5^{\circ}$ in diameter, respectively. All monkeys were trained for several months before the ${ }^{14} \mathrm{C}-\mathrm{DG}$ experiment to perform their tasks continuously for at least $1 \mathrm{~h}$ per day. On the day of the ${ }^{14} \mathrm{C}$-DG experiment, monkeys performed their tasks for the entire experimental period (45 min) without any breaks, and successful completion of each trial was 
A
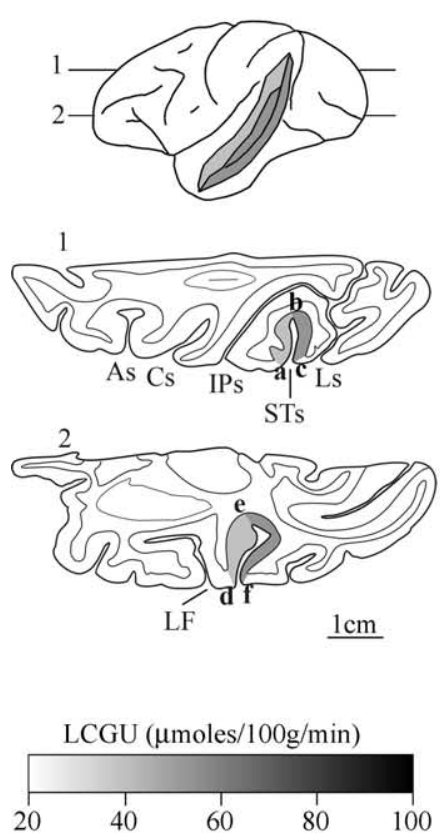

B

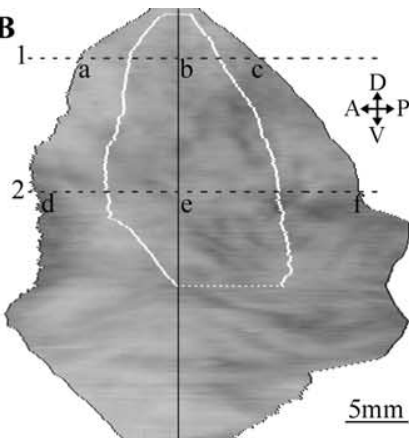

E

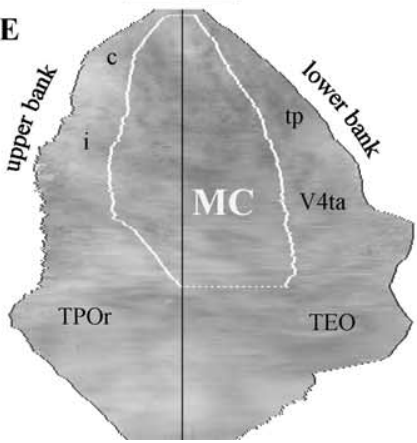

C

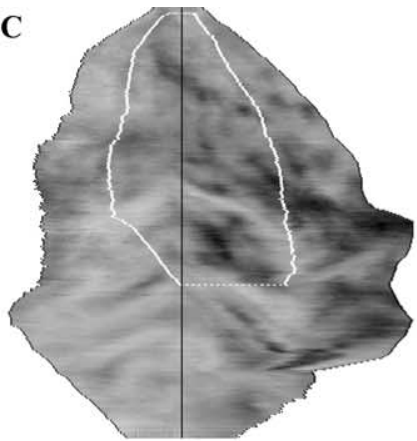

F

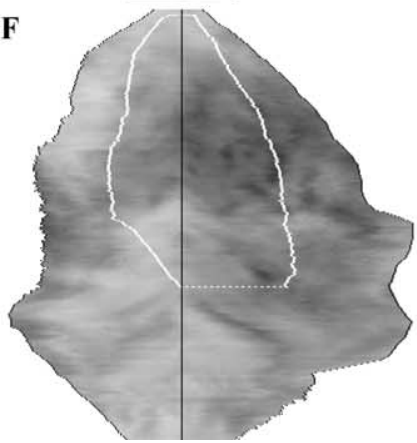

D
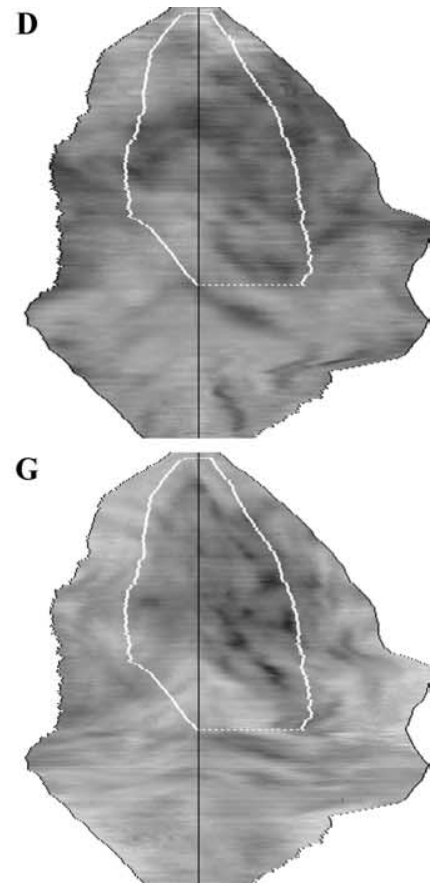

Figure 1. Quantitative 2D maps of metabolic activity in the STs. A, Lateral view of a monkey brain with an unfolded STs and a schematic illustration of two horizontal sections at the levels indicated in the brain drawing. In the sections, points $a$ and $d$ correspond to the crown of the upper bank, points $b$ and e correspond to the anterior tip of the fundus (used for alignment), and points $c$ and $f$ correspond to the crown of the lower bank. As, Arcuate sulcus; Cs, central sulcus; IPs, intraparietal sulcus; Ls, lunate sulcus. The grayscale bar indicates LCGU values (micromoles per $100 \mathrm{~g}$ per minute). $\boldsymbol{B}$, Average STs map from the two hemispheres of the Cf monkey. The MC is demarcated by white lines. A, Anterior; $D$, dorsal; $P$, posterior; $V$, ventral. Letters a-f correspond to those in $\boldsymbol{A}$. $\boldsymbol{C}$, STs map from the monkey executing visually guided horizontal saccades. $\boldsymbol{D}$, STs map from the monkey executing visually guided oblique saccades. $\boldsymbol{E}$, Average STs map from the two hemispheres of the Cd monkey. F, STs map from the monkey executing memory-guided horizontal saccades. G, STs map from the monkey executing memory-guided oblique saccades.

rewarded with water. The success rate remained approximately constant $(>90 \%)$ throughout the experiment.

The monkey performing "visually guided horizontal saccades" fixated a central visual target for $0.3-0.6 \mathrm{~s}$, then made $30^{\circ}$ rightward saccades and fixated for $0.3-0.6 \mathrm{~s}$ (intertrial intervals, $0.5-0.8 \mathrm{~s}$ ). The monkey performing "visually guided oblique saccades" executed $20^{\circ}$ saccades in the $135^{\circ}$ direction and fixated for $0.4-0.6 \mathrm{~s}$ (intertrial intervals, $0.3-0.5 \mathrm{~s}$ ). The monkey performing "memory-guided horizontal saccades" fixated straight ahead in total darkness after an auditory cue of $90 \mathrm{~Hz}$ and held its gaze for $0.4-0.7 \mathrm{~s}$ until a second auditory cue of $180 \mathrm{~Hz}$ signaled the execution of a memorized horizontal leftward saccade of $20^{\circ}$, followed by fixation for $0.4-0.7 \mathrm{~s}$ (intertrial intervals, $0.8-1.1 \mathrm{~s}$ ). The monkey performing "memory-guided oblique saccades" kept its eyes straight ahead in darkness for $0.5-0.7 \mathrm{~s}$ in response to an auditory cue of $90 \mathrm{~Hz}$, until a second auditory cue of $180 \mathrm{~Hz}$ commanded a memorized saccade of $25^{\circ}$ in amplitude and $135^{\circ}$ in direction, followed by fixation for $0.5-0.7 \mathrm{~s}$ (intertrial intervals, $0.5-0.7 \mathrm{~s}$ ). The fixation-control (Cf) monkey fixated a central visual target for $4 \mathrm{~s}$ per trial (intertrial intervals, $0.2-0.3 \mathrm{~s}$ ). The control monkey in the dark (Cd) was exposed to the same auditory stimuli as the monkeys performing memorized saccades and received reward at random intervals. In all tasks, parameters were chosen so that the total number of the stimulus-triggered saccades would be approximately equal. The saccades that brought the eyes back to the fixation point and the distribution of end points of all eye movements executed by the six monkeys have been presented previously (Moschovakis et al., 2004; Bakola et al., 2006).

${ }^{14} \mathrm{C}-\mathrm{DG}$ procedure. The ${ }^{14} \mathrm{C}-\mathrm{DG}$ experiment followed the procedure reported previously (Bakola et al., 2006). The tracer was injected intravenously $5 \mathrm{~min}$ after initiation of task performance, and arterial blood samples were drawn for the next $45 \mathrm{~min}$ as per the original description of the method (Sokoloff et al., 1977). Plasma glucose levels $(70-85 \mathrm{mg} / \mathrm{dl}$ ) as well as blood pressure, hematocrit, and blood gases ranged within normal values in all monkeys and remained constant for the duration of the ${ }^{14} \mathrm{C}$-DG experiment (Kennedy et al., 1978). Local cerebral glucose utilization (LCGU) values (in micromoles per $100 \mathrm{~g}$ per minute) were calculated from the original operational equation (Sokoloff et al., 1977). To cover the full extent of the STs, $\sim 1700$ serial horizontal sections of 20 $\mu \mathrm{m}$ thickness were cut in each hemisphere (Fig. $1 A$ ). For each horizontal section, a data array was obtained by sampling LCGU values (at a resolution of $54 \mu \mathrm{m} / \mathrm{pixel}$ ) along the rostrocaudal extent of the STs. At every five adjacent sections, data arrays were averaged and plotted to produce the two-dimensional (2D) maps of activity. The anterior tip of the floor of the STs was used for the alignment of adjacent sections. LCGU values were normalized by averaging the nonaffected gray-matter values in all hemispheres. The statistical significance of differences between LCGU values was tested with the Student's $t$ test. Because LCGU values of homonymous areas in opposite hemispheres of resting monkeys can differ by up to $7 \%$ (Savaki et al., 1993), only differences higher than $10 \%$ were considered significant.

Histological procedures. One section every $500 \mu \mathrm{m}$ was stained with thionine for the identification of the cytoarchitectonic borders of the MC. Our 20- $\mu$ m-thick sections of nonperfused tissue did not allow for myelin or SMI-32 immunostaining. The MC, which we identified in the caudal STs, extended from approximately halfway in the lower bank along the floor and into the upper bank and primarily corresponds to areas OAa and PGa described by Seltzer and Pandya (1978). It was characterized by the presence of a prominent layer VI, clearly distinct from the relatively poor layer V (Seltzer and Pandya, 1978; Hof and Morrison, 1995). We used the cytoarchitectonic borders of the MC, surface landmarks, and previously reported maps (Gattass and Gross, 1981; Desimone and Ungerleider, 1986; Boussaoud et al., 1990, 1991; Cusick et al., 1995; Lewis and Van Essen, 2000) to identify the STs areas we analyzed.

Geometric normalization of the STs maps. In all STs maps, the sectionby-section distances between (1) the anterior crown of the STs and the anterior cytoarchitectonic border of the MC, (2) the latter and the anterior tip of the floor of STs (point of alignment), (3) the latter and the posterior cytoarchitectonic borders of the MC, (4) the latter and the posterior crown of the STs, and (5) the dorsalmost and ventralmost tips of the STs were measured. The average of these measures was computed to produce a reference STs map of landmarks (Fig. $1 B-G$, overlay). In- 
Table 1. Metabolic effects in cortical areas of STs

\begin{tabular}{|c|c|c|c|c|c|c|c|}
\hline \multirow[b]{2}{*}{$\operatorname{Area}(n)$} & \multirow{2}{*}{$\frac{\text { Control, dark (Cd) }}{\mathrm{LCGU} \pm \mathrm{SD}}$} & \multicolumn{2}{|c|}{ Saccades, dark (Sd) } & \multicolumn{2}{|c|}{ Control, fixation (Cf) } & \multicolumn{2}{|c|}{ Saccades, light (SI) } \\
\hline & & $\mathrm{LCGU} \pm \mathrm{SD}$ & $\% \mathrm{Cd}^{a}$ & $\mathrm{LCGU} \pm \mathrm{SD}$ & $\% C d^{b}$ & $\mathrm{LCGU} \pm \mathrm{SD}$ & $\% \mathrm{Cf}$ \\
\hline V5/MT (72) & $54 \pm 3$ & $68 \pm 4$ & 26 & $57 \pm 2$ & 6 & $67 \pm 4$ & 18 \\
\hline $\operatorname{MTf}(25)$ & $53 \pm 3$ & $67 \pm 6$ & 26 & $61 \pm 5$ & 15 & $74 \pm 3$ & 21 \\
\hline MST (50) & $50 \pm 3$ & $60 \pm 4$ & 20 & $54 \pm 4$ & 8 & $66 \pm 4$ & 22 \\
\hline FST (40) & $46 \pm 4$ & $48 \pm 4$ & 4 & $56 \pm 5$ & 22 & $64 \pm 8$ & 14 \\
\hline TPOC (50) & $42 \pm 6$ & $42 \pm 5$ & 0 & $45 \pm 3$ & 7 & $49 \pm 5$ & 9 \\
\hline TP0i (50) & $42 \pm 6$ & $43 \pm 4$ & 2 & $45 \pm 4$ & 7 & $53 \pm 7$ & 18 \\
\hline TPOr (110) & $45 \pm 3$ & $47 \pm 4$ & 4 & $47 \pm 3$ & 4 & $50 \pm 2$ & 6 \\
\hline V4tp (145) & $51 \pm 4$ & $59 \pm 6$ & 16 & $54 \pm 4$ & 6 & $64 \pm 6$ & 19 \\
\hline V4ta (75) & $48 \pm 3$ & $54 \pm 6$ & 13 & $59 \pm 4$ & 23 & $67 \pm 8$ & 14 \\
\hline TEO (50) & $50 \pm 3$ & $53 \pm 3$ & 6 & $59 \pm 3$ & 18 & $64 \pm 4$ & 8 \\
\hline
\end{tabular}

$n$ represents the number of sets of five adjacent horizontal sections used to obtain the mean LCGU values for each region. (d and (f values represent the average $L C G U$ (in micromoles per $100 \mathrm{~g}$ per minute) from the two hemispheres of each control monkey. Sd and SI values represent the average LCGU from the two monkeys executing memorized and visual saccades, respectively. \% Cf is the percentage differences between SI and Cf values Calculated as (SI - Cf)/Cf $\times 100$. Values in bold indicate statistically significant differences by the Student's unpaired $t$ test at the level of $p<0.001$.

${ }^{a} \% \mathrm{Cd}$ is the percentage differences between $\mathrm{Sd}$ and $\mathrm{Cd}$ calculated as $(\mathrm{Sd}-\mathrm{Cd}) / \mathrm{Cd} \times 100$.

${ }^{b} \% \mathrm{Cd}$ is the percentage differences between $\mathrm{Cf}$ and $\mathrm{Cd}$ calculated as ( $\left.\mathrm{Cf}-\mathrm{Cd}\right) / \mathrm{Cd} \times 100$.

dividual ${ }^{14} \mathrm{C}-\mathrm{DG}$ and histological maps were linearly transformed in Matlab (MathWorks, Natick, MA) to match the reference map. This allowed us to overlay functional and cytoarchitectonic maps from all monkeys.

\section{Results}

\section{Visually guided saccades}

The monkey rewarded for performing visually guided horizontal saccades executed 191 such saccades during the critical first 10 min of the ${ }^{14} \mathrm{C}$-DG experiment, within an oculomotor space $5 \times$ $5^{\circ}$ centered on the peripheral target (saccade density, 7.6/degree $^{2}$ ). Because the landing area of saccades executed in the absence of targets (the memory-guided ones, described below) was more widespread, we also counted the number of saccades within a larger window $\left(10 \times 10^{\circ}\right)$. The number of saccades that were executed by the first monkey (visually guided horizontal ones) during the critical first 10 min of the ${ }^{14} \mathrm{C}-\mathrm{DG}$ experiment and that were contained within such an enlarged sampling window equaled 212 (saccade density, 2.12/degree ${ }^{2}$ ). This monkey also executed small $\left(<3^{\circ}\right)$ eye movements around the fixation window (density, 3.9/degree ${ }^{2}$ ) and few saccades in other directions and amplitudes (average density, 0.02/degree ${ }^{2}$ ). The monkey rewarded for performing visually guided oblique saccades executed 138 of them (density, 5.5/degree ${ }^{2}$; window, $5 \times 5^{\circ}$ ) during the critical first $10 \mathrm{~min}$ of the ${ }^{14} \mathrm{C}$-DG experiment. Here again, additional saccades landed near this window such that we counted 182 in a $10 \times 10^{\circ}$ window (saccade density, $1.82 /$ degree $^{2}$ ). Small $\left(<3^{\circ}\right)$ eye movements had a density of $3.4 /$ degree $^{2}$, whereas saccades of other amplitudes and directions had an average density of $0.1 /$ degree $^{2}$. The fixation-control monkey fixated the visual target for $75 \%$ of the time during the critical period. Small $\left(<3^{\circ}\right)$ movements around the fixation point had a density of $7.2 / \mathrm{de}-$ gree $^{2}$, whereas the average density of saccades executed outside the fixation window was $0.01 /$ degree $^{2}$.

Because areas V5/MT and MST are known to represent the contralateral half of visual space (Dubner and Zeki, 1971; Desimone and Ungerleider, 1986; Maunsell and Van Essen, 1987), we analyzed the STs contralateral to the saccades executed by the animal. Figure 1 provides high-resolution 2D quantitative LCGU maps of the STs reconstructed from serial horizontal sections through the entire extent of the sulcus. Images corresponding to right hemispheres are shown after reflection, so that the upper bank is always shown on the left side and the lower bank is always shown on the right side of the reconstructions. Functional (LCGU) and anatomical (cytoarchitectonic) reconstructions are superimposed. The surface area of the MC we identified was 200 $\mathrm{mm}^{2}$ and compares well with that illustrated in previous studies [125 mm ${ }^{2}$ (Cusick et al., 1995); $175 \mathrm{~mm}^{2}$ (Desimone and Ungerleider, 1986)]. Areas V5/MT in the posterior bank, MST in the anterior bank, and FST in the fundus of the STs were activated in both monkeys executing Sl (Fig. 1C,D; Table 1) compared with Cf (Fig. $1 \mathrm{~B}$ ). The enhancement of glucose consumption (range, 14$22 \%$ higher than the fixation control) was comparable to that induced by visually guided saccades in the SC, FEF, and LIP (range, 12-31\%) and higher than that induced in the SEF (411\%) (Moschovakis et al., 2001, 2004; Bakola et al., 2006). The $\mathrm{V} 5 / \mathrm{MT}$ activation included its ventralmost posterior part, which corresponds to area MT-foveal (MTf) and represents central vision (Gattass and Gross, 1981). Moreover, visually guided saccades induced activation of the intermediate temporal-parietaloccipital (TPOi) area anterior to the MST and posterior V4t (V4tp) and the anterior V4t (V4ta) posterior to MT (Fig. 1C,D; Table 1).

\section{Memory-guided saccades in complete darkness}

The monkey rewarded for performing acoustically triggered horizontal saccades in the dark executed 210 saccades from the central to the peripheral memorized location within a $10 \times 10^{\circ}$ sampling window (saccade density, 2.1/degree ${ }^{2}$ ) during the critical first $10 \mathrm{~min}$ of the ${ }^{14} \mathrm{C}$-DG experiment. Use of this window was dictated by the fact that, as expected of saccades executed in the absence of visual targets, these were more widely distributed than those made to visual targets. Small $\left(<3^{\circ}\right)$ eye movements had a density of $0.2 /$ degree $^{2}$, and saccades outside these regions had a density of $0.1 /$ degree $^{2}$. The monkey rewarded for performing oblique saccades in the dark executed 217 saccades (density, 2.2/ degree $^{2}$; window, $10 \times 10^{\circ}$ ) during the critical first 10 min of the ${ }^{14} \mathrm{C}$-DG experiment. Small $\left(<3^{\circ}\right)$ eye movements had a density of $0.6 /$ degree $^{2}$, and saccades outside these regions had a density of $0.03 /$ degree $^{2}$. Saccades executed by the control in the dark monkey were almost evenly distributed throughout its oculomotor space, with an average density of $0.04 /$ degree $^{2}$ in the central visual field and $0.03 /$ degree $^{2}$ in the peripheral field.

As in the monkeys executing visually guided saccades, areas V5/MT, MTf, MST, V4tp, and V4ta were activated in both monkeys executing memory-guided saccades (Fig. $1 F, G$; Table 1) compared with the $\mathrm{Cd}$ monkey (Fig. $1 E$ ). In contrast to the monkeys executing visually guided saccades, areas FST and TPOi were 

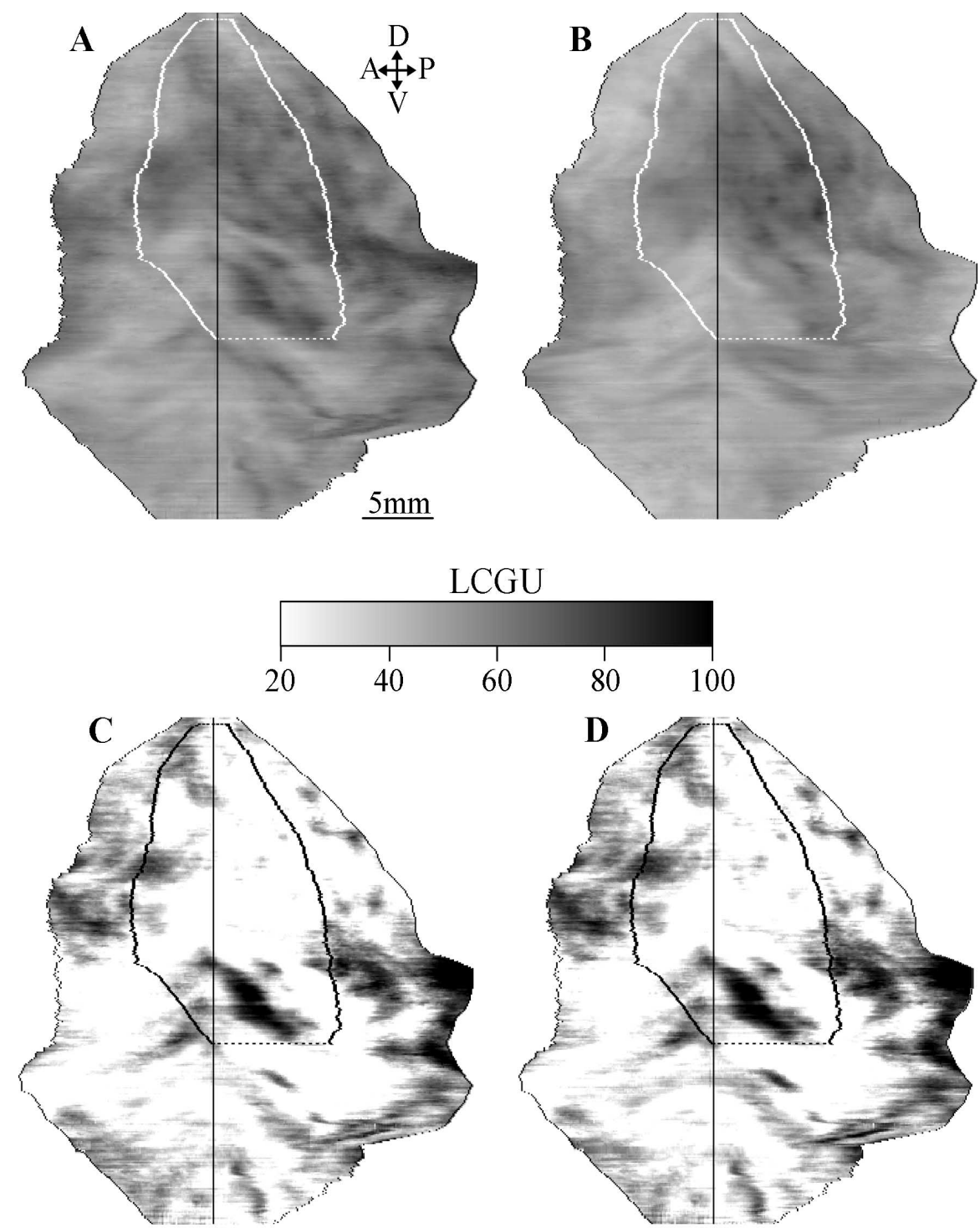

LCGU

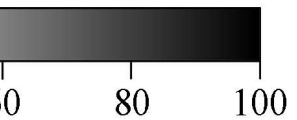

D
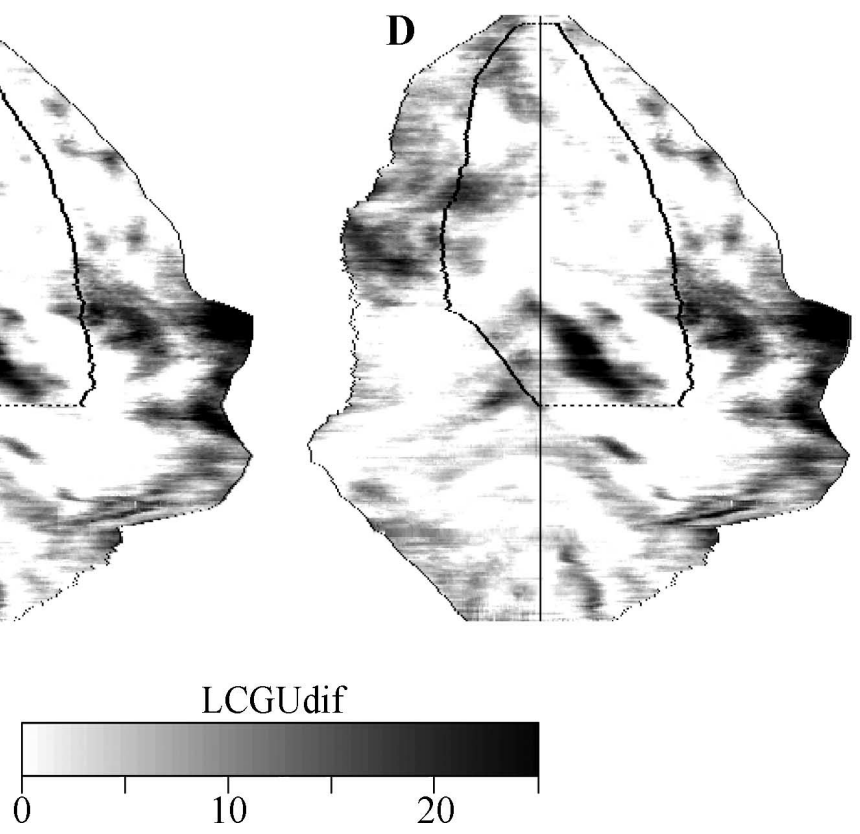

Figure 2. $A$, An averaged quantitative map showing metabolic activity in the STs from the two animals executing SI. A, Anterior; D, dorsal; $P$, posterior; $V$, ventral. $B$, Average STs map of metabolic activity in the monkeys executing Sd. The grayscale bar indicates LCGU values. C, Subtraction of map B from map A. D, This map is similar to the one shown in $\boldsymbol{C}$, except that the individual maps obtained from different monkeys were weighted to compensate for differences in oculomotor performance (number of saccades) before averaging and subtraction. The grayscale bar indicates LCGU differences (LCGUdif) in micromoles per $100 \mathrm{~g}$ per minute.

not activated in the monkeys executing memory-guided saccades (Table 1).

\section{Comparison of regions activated for visually and memory-guided saccades}

To better compare the effects of visually and memory-guided saccades, we generated two average quantitative maps of metabolic activity. The first one was produced by averaging the STs maps of the two monkeys executing visually guided saccades (Fig. $2 \mathrm{~A}$ ), and the second one was produced by averaging the corresponding maps of the two monkeys executing memory-guided saccades (Fig. 2 B). No activity remained in areas V5/MT, MST, and most of V4t when the latter was subtracted from the former (Fig. 2C), which implies that these areas are related to both visually and memory-guided saccades. Conversely, regions that remained activated include caudal parts of V4ta and temporal-occipital (TEO) areas, which are related to visual fixation (Table 1), and rostral FST and TPOi related to visually rather than memory-guided saccades (Fig. 2C).

Conceivably, differences in the activation patterns we observed could be partly attributable to differences in the oculomotor performance (number of saccades) of our monkeys during the critical first 10 min of the ${ }^{14} \mathrm{C}$-DG experiment. The number of saccades to the visual target (contained within an oculomotor space $10 \times$ $10^{\circ}$ ) was 212 and 182 for the two monkeys executing Sl. The number of saccades to the memorized target location (contained within the same $10 \times 10^{\circ}$ window and the same critical first $10 \mathrm{~min}$ of the experiment) was 210 and 217 for the two monkeys executing memorized saccades. To correct for such performance differences, we normalized the activity patterns observed in different monkeys to that observed in the monkey with the fewest number of saccades. To this end, we divided the LCGU values contained in the metabolic map of each monkey by the number of saccades it executed (to obtain an estimate of glucose consumption per pixel per movement) and multiplied it by the number of saccades (182) executed by the monkey with the poorest performance (oblique saccades to visual targets). Figure $2 D$ illustrates the quantitative map of metabolic activity obtained after averaging the weighted STs maps of the two monkeys executing visually guided saccades and subtracting the average map obtained from the weighted STs maps of the two monkeys executing memory-guided saccades. The similarity of the activity patterns shown in Figure 2, $C$ and $D$, implies that relatively small differences in oculomotor performance (e.g., the number of saccades executed by our monkeys) did not contribute appreciably to the STs activity patterns we observed.

Figure 3 illustrates the spatial relationship of STs regions activated in the two saccade-guidance conditions. First, the geometrically normalized STs functional maps of the two monkeys executing visually guided saccades were averaged, and all pixels with LCGU values higher (by $\geq 10 \%$ ) than those of the Cf were colorcoded red. The resulting map (Fig. $3 A$ ) was superimposed on the average STs map of the two Sd monkeys, which was generated by using the same $10 \%$ threshold over the Cd but color-coded blue (Fig. $3 B$ ). The region of overlap (red + blue $=$ violet $)$ demonstrates that approximately one-third of the neural space devoted 
to visually guided saccades (corresponding to areas V5/MT, MST, and V4t) also participates in memory-guided saccades in complete darkness (Fig. 3C). Figure 3 indicates that the lateral part of MST, which is adjacent and anterior to MTf and may correspond to part of the lateral MST (MSTl), is activated for both visually and memory-guided saccades whereas a more dorsal subregion, possibly corresponding to the dorsal MST, is activated mainly for saccades to visual targets. The former (MSTl) is causally associated with pursuit eye movements as shown by lesion (Dursteler and Wurtz, 1988) and microstimulation (Komatsu and Wurtz, 1989) studies.

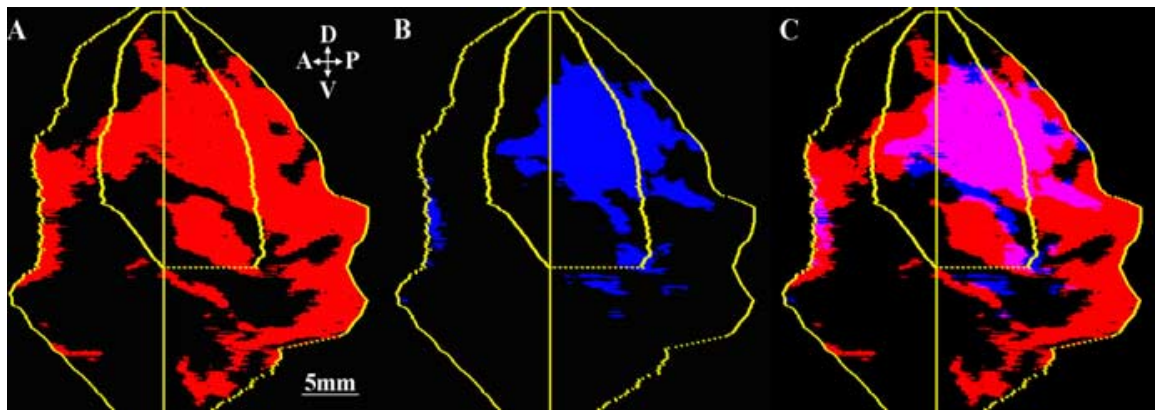

Figure 3. $\quad A$, Averaged qualitative STs map from the two SI monkeys. Pixels with LCGU values higher than those of the control (Cf) by $>10 \%$ are shown in red. A, Anterior; D, dorsal; P, posterior; V, ventral. $\boldsymbol{B}$, Averaged STs map from the two Sd monkeys. Pixels with $L C G U$ values higher than the control (Cd) by $>10 \%$ are color-coded blue. $C$, A superimposition of $A$ and $\boldsymbol{B}$. The region of overlap (violet) results from the presence of both red and blue.

Fixation activated areas are the MTf, FST, V4ta, and TEO (Table 1, Cf, \%Cd). It should be noted, however, that the fixation of visual targets involves not only exposure to sensory stimulation but also a considerable number of small eye movements executed around the fixation target.

\section{Discussion}

This study is the first to provide high-resolution quantitative functional images of the location and extent of STs cortical areas engaged in saccades to visual targets and saccades to memorized locations in the dark. We demonstrate that areas V5/MT and MST, traditionally thought to process visuomotion signals for smooth-pursuit eye movements, receive and/or process extraretinal oculomotor signals when monkeys execute visually and memory-guided saccades.

Our data demonstrate that areas FST and TPOi are activated only for saccades to visual targets and not for saccades to memorized locations. The region anatomically defined as TPO (Seltzer and Pandya, 1978) corresponds to the superior temporal polysensory area (Bruce et al., 1981) and derives its input from the $\mathrm{MC}$ in the dorsal pathway and from the anterior inferior temporal area in the ventral pathway (Boussaoud et al., 1990). We have used the three caudal-to-rostral subdivisions, TPOc (caudal), TPOi, and TPOr (rostral), as suggested previously (Cusick et al., 1995). Of interest is that the only subdivision found to be activated by visual saccades in our study was TPOi that, as with areas V5/MT and MST, receives convergent projections from oculomotor areas such as the dorsal and ventral prearcuate and the posterior parietal (Cusick et al., 1995). Consistent with our findings, TPO has been implicated in the control of visually guided saccades, because its damage prolongs their latency (Scalaidhe et al., 1995). On the other hand, the anterior/ventral part of STs, which has been associated with integration of animate form motion and location (Jellema et al., 2004), remained unaffected in our study.

Our data also demonstrate that both saccades to visual targets and to memorized locations activated area $\mathrm{V} 4 \mathrm{t}$, located posterior to MT in the STs. In agreement with our findings, there are reports of presaccadic/perisaccadic enhancement in V4 cell activity (Fischer and Boch, 1981; Moore et al., 1998). The subdivisions of area V4t (V4ta and V4tp) that we use are based on the STs surface landmarks and our MC cytoarchitectonic borders, according to the anatomical maps of Lewis and Van Essen (2000). Both of these subdivisions were activated for both visual and memorized saccades, with the only difference being that V4ta was also activated for fixation. Our visual fixation effects in V4ta and TEO are

in agreement with the central visual field representation demonstrated in these regions in STs (Boussaoud et al., 1991). Compatible with the fixation-induced activation of V4ta and TEO is the report that $41 \%$ of TEO and $34 \%$ of V4 neurons were activated during a spot-fixation task (Watanabe, 1996). Actually, during fixation when the foveal visual cortices are excited, apart from V4ta and TEO, the only other areas we found activated were FST and MTf, which are thought to represent central vision (Gattass and Gross, 1981; Desimone and Ungerleider, 1986; Boussaoud et al., 1990,1991) and help maintain fixation (Komatsu and Wurtz, 1989)

In our study, all three areas that comprise the MC, V5/MT, MST, and FST are activated for saccades to visual targets, whereas only V5/MT and MST are activated for saccades to memorized locations in the dark. In addition to directionally selective neurons displaying sensory (retinal) discharges, MST and, to a smaller degree, MTf contain neurons displaying extraretinal signals associated with the maintenance of pursuit (Newsome et al., 1988; Ferrera and Lisberger, 1997). Nonetheless, the visual and oculomotor components of MC neuronal discharge have always been assigned a role in smooth pursuit rather than rapid eye movements. Our data demonstrate that the MC is activated for saccades as well. Thus, they are in agreement with previous imaging studies that have consistently shown activation of the superior temporal cortex for visually guided (Sweeney et al., 1996; Petit and Haxby, 1999; Koyama et al., 2004) and memory-guided (Anderson et al., 1994; Luna et al., 1998; Ozyurt et al., 2006) saccades. However, it should be noted that we provided no visual stimulation during the execution of the memory-guided task, whereas previous studies relied on retinal stimulation, albeit minimally, because of the presence of visual targets extinguished before movement onset. Also, our method provides higher sampling resolution (20 $\mu \mathrm{m}$ sections) and histological confirmation of the anatomical borders of the MC. Our study is in better agreement with a more recent, well controlled functional magnetic resonance imaging study (Baker et al., 2006) of monkeys executing visually guided saccades, showing activation of the superior temporal cortex [including areas MT, MST, FST, STP (superior temporal polysensory), and V4]. In this study, the robust activation of the MT/MST/FST complex was considered unexpected and was attributed to the visual motion induced on the retina by saccades. Indeed, motion signals accompanying saccades are as effective in eliciting responses from V1 neurons as the motion of objects in the visual field (Wurtz, 1969). Consequently, part of the visually guided saccade-related activation of the MC we observed could be because of the shear of the visual scene on the 
retina. Such a mechanism is doubtful for areas V5/MT and MST, because they contain neurons that process oppositely directed motion signals that could annul each other (Thiele et al., 2002). More importantly, such a mechanism cannot explain the herein documented activation of areas V5/MT and MST for memoryguided saccades in the dark, which was equally robust to that for visually guided saccades, and could only reflect the presence of extraretinal saccade-related signals. To our knowledge, this is the first study to conclusively demonstrate a correlation between the execution of saccadic eye movements and the activity in the pursuit-related areas V5/MT and MST.

\section{References}

Anderson TJ, Jenkins IH, Brooks DJ, Hawken MB, Frackowiak RS, Kennard C (1994) Cortical control of saccades and fixation in man. A PET study. Brain 117:1073-1084.

Baker JT, Patel GH, Corbetta M, Snyder LH (2006) Distribution of activity across the monkey cerebral cortical surface, thalamus and midbrain during rapid, visually guided saccades. Cereb Cortex 16:447-459.

Bakola S, Gregoriou GG, Moschovakis AK, Savaki HE (2006) Functional imaging of the intraparietal cortex during saccades to visual and memorized targets. NeuroImage 31:1637-1649.

Boussaoud D, Ungerleider LG, Desimone R (1990) Pathways for motion analysis: cortical connections of the medial superior temporal and fundus of the superior temporal visual areas in the macaque. J Comp Neurol 296:462-495.

Boussaoud D, Desimone R, Ungerleider LG (1991) Visual topography of area TEO in the macaque. J Comp Neurol 306:554-575.

Bremmer F, Distler C, Hoffmann KP (1997) Eye-position effects in monkey cortex. II: Pursuit and fixation related activity in posterior parietal areas LIP and 7A. J Neurophysiol 77:962-977.

Bruce CJ, Desimone R, Gross CG (1981) Visual properties of neurons in a polysensory area in superior temporal sulcus of the macaque. J Neurophysiol 46:369-384.

Cusick CG, Seltzer B, Cola M, Griggs E (1995) Chemoarchitectonics and corticocortical terminations within the superior temporal sulcus of the rhesus monkey: evidence for subdivisions of superior temporal polysensory cortex. J Comp Neurol 360:513-535.

Desimone R, Ungerleider LG (1986) Multiple visual areas in the caudal superior temporal sulcus of the macaque. J Comp Neurol 248:164-189.

Dubner R, Zeki SM (1971) Response properties and receptive fields of cells in an anatomically defined region of the superior temporal sulcus in the monkey. Brain Res 34:528-532.

Dursteler MR, Wurtz RH (1988) Pursuit and optokinetic deficits following chemical lesions of cortical areas MT and MST. J Neurophysiol 60:940-965.

Ferrera VP, Lisberger SG (1997) Neuronal responses in visual areas MT and MST during smooth pursuit target selection. J Neurophysiol 78:1433-1446.

Fischer B, Boch R (1981) Enhanced activation of neurons in prelunate cortex before visually guided saccades of trained rhesus monkeys. Exp Brain Res 44:129-137.

Gattass R, Gross CG (1981) Visual topography of striate projection zone (MT) in posterior superior temporal sulcus of the macaque. J Neurophysiol 46:621-628.

Heinen SJ (1995) Single neuron activity in the dorsomedial frontal cortex during smooth pursuit eye movements. Exp Brain Res 104:357-361.

Hof PR, Morrison JH (1995) Neurofilament protein defines regional patterns of cortical organization in the macaque monkey visual system: a quantitative immunohistochemical analysis. J Comp Neurol 352:161-186.

Jellema T, Maassen G, Perrett DI (2004) Single cell integration of animate form, motion and location in the superior temporal cortex of the macaque monkey. Cereb Cortex 2004:781-790.

Kennedy C, Sakurada O, Shinohara M, Jehle J, Sokoloff L (1978) Local cerebral glucose utilization in the normal conscious macaque monkey. Ann Neurol 4:293-301.
Komatsu H, Wurtz RH (1989) Modulation of pursuit eye movements by stimulation of cortical areas MT and MST. J Neurophysiol 62:31-47.

Koyama M, Hasegawa I, Osada T, Adachi Y, Nakahara K, Miyashita Y (2004) Functional magnetic resonance imaging of macaque monkeys performing visually guided saccade tasks: comparison of cortical eye fields with humans. Neuron 41:795-807.

Krauzlis RJ (2004) Recasting the smooth pursuit eye movement system. J Neurophysiol 91:591-603.

Krauzlis RJ, Basso MA, Wurtz RH (1997) Shared motor error for multiple eye movements. Science 276:1693-1695.

Lewis JW, Van Essen DC (2000) Mapping of architectonic subdivisions in the macaque monkey, with emphasis on parieto-occipital cortex. J Comp Neurol 428:79-111.

Luna B, Thulborn KR, Strojwas MH, McCurtain BJ, Berman RA, Genovese CR, Sweeney JA (1998) Dorsal cortical regions subserving visually guided saccades in humans: an fMRI study. Cereb Cortex 8:40-47.

Maunsell JH, Van Essen DC (1983) Functional properties of neurons in middle temporal visual area of the macaque monkey. I. Selectivity for stimulus direction, speed, and orientation. J Neurophysiol 49:1127-1147.

Maunsell JH, Van Essen DC (1987) Topographic organization of the middle temporal visual area in the macaque monkey: representational biases and the relationship to callosal connections and myeloarchitectonic boundaries. J Comp Neurol 266:535-555.

Moore T, Tolias AS, Schiller PH (1998) Visual representations during sacadic eye movements. Proc Natl Acad Sci USA 95:8981-8984.

Moschovakis AK, Gregoriou GG, Savaki HE (2001) Functional imaging of the primate superior colliculus during saccades to visual targets. Nat Neurosci 4:1026-1031.

Moschovakis AK, Gregoriou GG, Ugolini G, Doldan M, Graf W, Guldin W, Hadjidimitrakis K, Savaki HE (2004) Oculomotor areas of the primate frontal lobes: a transneuronal transfer of rabies virus and [14C]-2deoxyglucose functional imaging study. J Neurosci 24:5726-5740.

Newsome WT, Wurtz RH, Komatsu H (1988) Relation of cortical areas MT and MST to pursuit eye movements. II. Differentiation of retinal from extraretinal inputs. J Neurophysiol 5:825-840.

Ozyurt J, Rutschmann RM, Greenlee MW (2006) Cortical activation during memory-guided saccades. NeuroReport 17:1005-1009.

Petit L, Haxby JV (1999) Functional anatomy of pursuit eye movements in humans as revealed by fMRI. J Neurophysiol 82:463-471.

Savaki HE, Kennedy C, Sokoloff L, Mishkin M (1993) Visually guided reaching with the forelimb contralateral to a "blind" hemisphere: a metabolic mapping study in monkeys. J Neurosci 13:2772-2789.

Scalaidhe SP, Albright TD, Rodman HR, Gross CG (1995) Effects of superior temporal polysensory area lesions on eye movements in the macaque monkey. J Neurophysiol 73:1-19.

Seltzer B, Pandya DN (1978) Afferent cortical connections and architectonics of the superior temporal sulcus and surrounding cortex in the rhesus monkey. Brain Res 149:1-24.

Sokoloff L, Reivich M, Kennedy C, Des Rosiers MH, Patlak CS, Pettigrew KS, Sakurada O, Shinohara M (1977) The $\left[{ }^{14} \mathrm{C}\right]$-deoxyglucose method for the measurement of local cerebral glucose utilization: theory, procedure, and normal values in the conscious and anesthetized albino rat. J Neurochem 28:879-916.

Sweeney JA, Mintun MA, Kwee S, Wiseman MB, Brown DL, Rosenberg DR, Carl JR (1996) Positron emission tomography study of voluntary saccadic eye movements and spatial working memory. J Neurophysiol $75: 454-468$

Tanaka M, Lisberger SG (2002) Role of arcuate frontal cortex of monkeys in smooth pursuit eye movements. I. Basic response properties to retinal image motion and position. J Neurophysiol 87:2684-2699.

Thiele A, Henning P, Kubischik M, Hoffmann K-P (2002) Neural mechanisms of saccadic suppression. Science 295:2460-2464.

Watanabe M (1996) Reward expectancy in primate prefrontal neurons. Nature 382:629-632.

Wurtz RH (1969) Comparison of effects of eye movements and stimulus movements on striate cortex neurons of the monkey. J Neurophysiol 32:987-994.

Zeki SM (1974) Functional organization of a visual area in the posterior bank of the superior temporal sulcus of the rhesus monkey. J Physiol (Lond) 236:549-573. 